\title{
Heterosis Studies for Seed Yield and Earliness in Intra-specific Hybrids of Ricebean [Vigna umbellata (Thunb.) Ohwi and Ohashi] an under Utilized Pulse
}

\author{
Neelam Bhardwaj*, Tanuja Kapoor and Sanchit Thakur
}

Department of Organic Agriculture, CSKHPKV, Palampur, India-176062

*Corresponding author

\begin{tabular}{|l|}
\hline Key w o r d s \\
Heterosis, \\
Ricebean, Vigna \\
umbellata, Hybrid \\
vigour
\end{tabular}

A B S T R A C T

A study was conducted in ricebean [Vigna umbellata (Thunb.) Ohwi and Ohashi] at Department of Organic Agriculture, CSKHPKV, Palampur to assess the extent of heterosis for eleven characters including grain yield per plant. Six lines and two testers were crossed in line $\times$ tester mating design to develop $12 \mathrm{~F} 1$ hybrids. The analysis of variance revealed considerable genetic differences among the genotypes. The variance due to parents was significant for all the traits except pods per cluster whereas variance due to crosses was significant all the traits under study. The variance due to parents vs hybrids was also highly significant for all the traits. Among the parents, RBHP-108 (11.95 g), RBHP43(11.94g), RBHP-36(11.33g), RBHP-107(11.22g) and RBHP-38(11.00g) were the highest yielders whereas among the hybrids, RBHP-36 x RBHP-900(14.78g), RBHP61x2007-2(14.42g) and RBHP-43x2007-2(12.67g) recorded highest grain yield per plant. Two crosses RBHP-36x2007-2(96 days) and RBHP-38x2007-2(96.33) were significantly early among all the crosses. Results indicated an appreciable amount of heterosis for all the traits under study and varied from character to character. Days to flowering, days to maturity and plant height showed significant negative heterotic effect over mid parent and better parent in 6 crosses viz., RBHP-36 $\times$ PRR-2007-2, RBHP-38 $\times$ PRR-2007-2, RBHP$38 \times$ RBHP-900, RBHP-43 × RBHP-900, RBHP-61 $\times$ RBHP-900 and RBHP-108 $x$ RBHP-900. Significant positive heterosis for grain yield was observed for 2 crosses viz; RBHP-36 × RBHP-900 and RBHP-61 x PRR-2007-2.

\section{Introduction}

Ricebean [Vigna umbellata (Thunb.) Ohwi and Ohashi] is one of the underutilized warm season annual vine legumes. Its seed contains $25 \%$ protein, $0.49 \%$ fat and $5 \%$ fibre. It is also rich in methionine and tryptophan as well as vitamins (thiamine, niacin, riboflavin and ascorbic acid) and restores soil fertility through biological nitrogen fixation (Ebert,
2014). Despite having all favourable traits, it is not much popular among the farmers due to the late maturity and indeterminate growth habit. A little improvement with respect to these traits can enhance the utility of this crop which can be done through selection of genotypes with desirable characters from the variation through recombination followed by selection. Though being self- pollinated, scope of exploitation of heterosis is limited in 
this crop however, the information on this aspect in $F_{1} s$ helps to identify the potential crosses for the development of varieties. Scope for the exploitation of hybrid vigour depends on the direction and magnitude of heterosis, biological feasibility and type of gene action involved. The information on heterosis will have a direct bearing on breeding methodology to be adopted for varietal improvement. Therefore, the present investigation was carried out to know the direction and magnitude of heterosis in ricebean.

\section{Materials and Methods}

Six indeterminate but high yielding genotypes of ricebean namely RBHP-36, RBHP-38, RBHP-43, RBHP-61, RBHP-107 and RBHP108 and two early maturing genotypes PRR2007-2 and RBHP-900 were selected for present study. Crosses were attempted in line $\mathrm{x}$ tester design and the resultant $12 \mathrm{~F}_{1}$ 's along with parents were evaluated in randomized block design with three replications. Each entry was sown in row of $2 \mathrm{~m}$ length with spacing $30 \times 10 \mathrm{~cm}$. The observations were recorded for eleven traits viz., days to $50 \%$ flowering, days to $75 \%$ maturity plant height (cm), number of branches per plant, pods per plant, number of clusters per plant, pods per cluster, seeds per pod, 100-seed weight (g), pod length $(\mathrm{cm})$ and seed yield per plant $(\mathrm{g})$. Heterosis expressed as per cent, was estimated for all the characters over mid parent (MP) and better parent (BP) as per standard procedure. Analysis was done as per the method given by Kempthorne (1957).

\section{Results and Discussion}

Analysis of variance for line $\mathrm{x}$ tester mating design with respect to parents (6 lines and two testers) and crosses revealed significant differences among crosses for all the yield traits studied (Table 1). Parents revealed significant differences for all the traits except pods per cluster. Further partitioning of variance of parents into lines, testers and lines vs testers indicated significant differences among lines for all the traits except pods per cluster. Testers also differed significantly for all the traits studied except branches per plant, pods per cluster, seeds per pod and 100-seed weight. The lines differed non-significantly from testers for branches per plant, clusters per plant and seeds per pod. Parents differed non-significantly from crosses for branches per plant and seeds per pod.

For grain yield, which is a complex character, only few crosses depicted conspicuous heterotic response over mid as well as better parent values. The range of heterosis over MP and BP was from $-10.07 \%$ to $121.00 \%$ and $28.72 \%$ to $72.45 \%$, respectively with the higher general magnitude of positive heterosis than the negative heterosis. Five crosses exhibited significant positive heterosis over mid parent while two crosses registered high significant positive heterosis over better parent. Among all the hybrids, RBHP-61 $\times$ PRR-2007-2 showed highest positive and significant heterosis over mid $(121.00 \%)$ as well as better (72.45\%) parent. Grain yield is polygenically controlled characters and depends on large number of other related characters. In the present study, significant positive heterosis in grain yield was found to be associated with number of branches per plant, pods per plant, number of clusters per plant and pods per cluster clearly indicated that heterosis for grain yield was through heterosis for individual yield components or additive or synergistic effects of the component characters. Significant positive heterotic effect for grain yield per plant over mid parent and better parent were also observed by Lakshmana et al., (2007) Sharma et al., (1998) and Vaidya et al., (2016) in ricebean. 
Since the main objective of the study was to incorporate earliness and determinate habit into the otherwise high yielding genotypes, hence for the developmental traits like days to maturity and plant height significant negative heterosis will be desirable. Out of the 12 cross combinations 5 crosses registered negative significant heterosis over mid parent while all crosses show significant negative heterosis over better parent for days to flowering. For days to maturity, 4 crosses exhibited significant negative heterosis over mid parent and 7 crosses exhibited significant negative heterosis over better parent. For plant height, as many as 9 crosses out of 12 exhibited significant negative heterosis over the better parent whereas 4 crosses showed significant negative heterosis over mid parent. Cross RBHP-38 $\times$ RBHP-900 depicted highest figure of negative heterosis $(-27.84 \%)$ while RBHP-36 × PRR-2007-2 showed highest value over the better parent $(-38.65 \%)$. Thamodharan et al., (2016) also conducted similar study to estimate the magnitude of economic heterosis for exploitation of hybrid vigour of crosses for higher yield and early maturity in blackgram and observed higher positive significant standard heterosis for 8 yield and yield attributing traits in positive direction and negative heterosis for two traits viz., days to 50 per cent flowering and days to maturity (Table 1-4).

Table.1 Analysis of variance for parents and hybrids

\begin{tabular}{|c|c|c|c|c|c|c|c|c|c|}
\hline Eileen center & & Replication & Parents & Lines & Testers & $\begin{array}{l}\text { Lines vs. } \\
\text { testers }\end{array}$ & Crosses & $\begin{array}{c}\text { Parents vs. } \\
\text { Hybrid }\end{array}$ & Error \\
\hline Traits & df & 2 & 7 & 5 & 1 & 1 & 11 & 1 & 38 \\
\hline $\begin{array}{l}\text { Days to } 50 \% \\
\text { flowering }\end{array}$ & & 3.51 & $389.75^{*}$ & $7.68 *$ & $228.16^{*}$ & $2461.68^{*}$ & $41.87 *$ & $319.225^{*}$ & 1.972 \\
\hline $\begin{array}{l}\text { Days to } 75 \% \\
\text { maturity }\end{array}$ & & 1.71 & $288.18^{*}$ & $59.68^{*}$ & $160.16^{*}$ & $1558.68 *$ & $427.57 *$ & $3.025^{*}$ & 5.102 \\
\hline Plant height & & 95.16 & $1265.73 *$ & $131.44 *$ & $505.81^{*}$ & $7697.09 *$ & $1251.85^{*}$ & $1289.284^{*}$ & 33.916 \\
\hline $\begin{array}{l}\text { Branches } \\
\text { per plant }\end{array}$ & & 0.07 & $0.37 *$ & $0.50^{*}$ & 0.060 & 0.045 & $0.46^{*}$ & 0.045 & 0.029 \\
\hline $\begin{array}{l}\text { Pods per } \\
\text { plant }\end{array}$ & & 0.64 & $95.77 *$ & $43.29 *$ & $168.540^{*}$ & $285.44^{*}$ & $150.49 *$ & $3.672 *$ & 1.851 \\
\hline $\begin{array}{l}\begin{array}{l}\text { Clusters per } \\
\text { plant }\end{array} \\
\end{array}$ & & 0.41 & $21.74 *$ & $11.83^{*}$ & 92.826* & 0.24 & $53.821 *$ & $41.391^{*}$ & 0.243 \\
\hline $\begin{array}{l}\text { Pods per } \\
\text { cluster }\end{array}$ & & 0.44 & 0.11 & 0.04 & 0.006 & $0.55^{*}$ & $1.006^{*}$ & $0.571^{*}$ & 0.059 \\
\hline $\begin{array}{l}\text { Seeds per } \\
\text { pod }\end{array}$ & & 0.14 & $3.46^{*}$ & $4.82 *$ & 0.060 & 0.05 & $1.945^{*}$ & $0.738 *$ & 0.234 \\
\hline $\begin{array}{l}100 \text { seed } \\
\text { weight }\end{array}$ & & 1.08 & $1.56^{*}$ & $0.81^{*}$ & 0.066 & $6.79 *$ & $1.717 *$ & $3.524 *$ & 0.136 \\
\hline $\begin{array}{l}\text { Pod length } \\
\text { (cm) }\end{array}$ & & 1.46 & $10.96^{*}$ & $1.09^{*}$ & $0.836^{*}$ & $70.44 *$ & $3.633^{*}$ & $1.080^{*}$ & 0.256 \\
\hline $\begin{array}{l}\text { Yield per } \\
\text { plant }\end{array}$ & & 36.31 & $21.84 *$ & $5.34 *$ & $7.990 *$ & $118.21^{*}$ & $15.322 *$ & $11.403^{*}$ & 1.801 \\
\hline
\end{tabular}


Table. 2 Estimates of heterosis for different traits in ricebean hybrids

\begin{tabular}{|l|l|l|l|l|l|l|}
\hline Crosses & \multicolumn{2}{l|}{ Days to flowering } & \multicolumn{2}{l|}{ Days to maturity } & \multicolumn{2}{l|}{ Plant height } \\
\hline & $\begin{array}{l}\text { Heterosis } \\
\text { over mid- } \\
\text { parent }(\%)\end{array}$ & $\begin{array}{l}\text { Heterosis over } \\
\text { better parent } \\
(\%)\end{array}$ & $\begin{array}{l}\text { Heterosis } \\
\text { over mid- } \\
\text { parent }(\%)\end{array}$ & $\begin{array}{l}\text { Heterosis } \\
\text { over better } \\
\text { parent }(\%)\end{array}$ & $\begin{array}{l}\text { Heterosis } \\
\text { over mid- } \\
\text { parent }(\%)\end{array}$ & $\begin{array}{l}\text { Heterosis } \\
\text { over better } \\
\text { parent }(\%)\end{array}$ \\
\hline RBHP-36 $\times$ PRR-2007-2 & 1.53 & $-16.74^{*}$ & $-9.15^{*}$ & $-20.66^{*}$ & $-18.73^{*}$ & $-38.65^{*}$ \\
\hline RBHP-36 $\times$ RBHP-900 & $3.50^{*}$ & $-7.11^{*}$ & $15.49^{*}$ & $5.79^{*}$ & 6.20 & $-12.08^{*}$ \\
\hline RBHP-38 $\times$ PRR-2007-2 & $3.05^{*}$ & $-15.77^{*}$ & $-6.32^{*}$ & $-16.47^{*}$ & -8.88 & $-28.15^{*}$ \\
\hline RBHP-38 $\times$ RBHP-900 & $-3.48^{*}$ & $-13.69^{*}$ & -2.78 & $-8.96^{*}$ & $-27.84^{*}$ & $-37.14^{*}$ \\
\hline RBHP-43 $\times$ PRR-2007-2 & $4.08^{*}$ & $-14.64^{*}$ & $4.09^{*}$ & $-6.47^{*}$ & $30.48^{*}$ & 0.62 \\
\hline RBHP-43 $\times$ RBHP-900 & $-6.29^{*}$ & $-15.90^{*}$ & $-6.54^{*}$ & $-11.76^{*}$ & 1.43 & $-13.92^{*}$ \\
\hline RBHP-61 $\times$ PRR-2007-2 & $8.95^{*}$ & $-10.50^{*}$ & $9.24^{*}$ & -1.19 & $32.67 *$ & 4.60 \\
\hline RBHP-61 $\times$ RBHP-900 & $-2.80^{*}$ & $-12.61^{*}$ & -1.10 & $-5.97 * *$ & -1.69 & $-14.37^{*}$ \\
\hline RBHP-107 $\times$ PRR-2007-2 & $6.33^{*}$ & $-13.22^{*}$ & $13.13^{*}$ & $4.02 *$ & $50.57^{*}$ & $20.36^{*}$ \\
\hline RBHP-107 $\times$ RBHP-900 & $-4.17^{*}$ & $-14.46^{*}$ & $29.28^{*}$ & $25.08^{*}$ & $-19.34^{*}$ & $-28.61^{*}$ \\
\hline RBHP-108 $\times$ PRR-2007-2 & $18.87^{*}$ & $-4.38^{*}$ & $10.36^{*}$ & -1.73 & $-20.69^{*}$ & $-38.11^{*}$ \\
\hline RBHP-108 $\times$ RBHP-900 & $-7.03^{*}$ & $-18.33^{*}$ & $-6.63^{*}$ & $-12.68^{*}$ & -6.24 & $-19.31^{*}$ \\
\hline
\end{tabular}

\begin{tabular}{|c|c|c|c|c|c|c|c|c|}
\hline \multirow[t]{2}{*}{ Crosses } & \multicolumn{2}{|c|}{ Branches per plant } & \multicolumn{2}{|c|}{ Pods per plant } & \multicolumn{2}{|c|}{ Clusters per plant } & \multicolumn{2}{|c|}{ Pods per cluster } \\
\hline & $\begin{array}{l}\text { Heterosis } \\
\text { over } \\
\text { mid- } \\
\text { parent } \\
(\%)\end{array}$ & $\begin{array}{l}\text { Heterosis } \\
\text { over } \\
\text { better } \\
\text { parent } \\
(\%)\end{array}$ & $\begin{array}{l}\text { Heterosis } \\
\text { over mid- } \\
\text { parent }(\%)\end{array}$ & $\begin{array}{l}\text { Heterosis } \\
\text { over } \\
\text { better } \\
\text { parent } \\
(\%)\end{array}$ & $\begin{array}{l}\text { Heterosis } \\
\text { over } \\
\text { mid- } \\
\text { parent } \\
(\%)\end{array}$ & $\begin{array}{l}\text { Heterosis } \\
\text { over } \\
\text { better } \\
\text { parent } \\
(\%)\end{array}$ & $\begin{array}{l}\text { Heterosis } \\
\text { over } \\
\text { mid- } \\
\text { parent } \\
(\%)\end{array}$ & $\begin{array}{l}\text { Heterosis } \\
\text { over } \\
\text { better } \\
\text { parent } \\
(\%)\end{array}$ \\
\hline $\begin{array}{l}\text { RBHP-36 × PRR-2007- } \\
2\end{array}$ & $-21.57 *$ & $-23.08 *$ & -0.55 & $-16.77 *$ & $15.23 *$ & $8.10 *$ & -1.49 & -5.71 \\
\hline RBHP-36 × RBHP-900 & $50.00 *$ & $38.46^{*}$ & $26.79 *$ & $23.96^{*}$ & $25.00 *$ & $5.96 *$ & $42.73 *$ & $34.57 *$ \\
\hline $\begin{array}{l}\text { RBHP-38 × PRR-2007- } \\
2\end{array}$ & $47.83 *$ & $36.00^{*}$ & 1.87 & $-13.16^{*}$ & $45.99 *$ & $31.43^{*}$ & 3.70 & -1.41 \\
\hline RBHP-38 × RBHP-900 & -2.33 & -4.55 & $-19.38 *$ & $-19.40 *$ & $-27.42 *$ & $-36.09 *$ & 11.28 & 4.23 \\
\hline $\begin{array}{l}\text { RBHP-43 × PRR-2007- } \\
2\end{array}$ & $60.98 *$ & $32.00 *$ & $48.13^{*}$ & $27.98^{*}$ & $47.60^{*}$ & $28.80 *$ & 10.29 & 4.17 \\
\hline RBHP-43 × RBHP-900 & $78.95 *$ & $54.55^{*}$ & $-7.76^{*}$ & $-9.20 *$ & $-24.21 *$ & $-31.13^{*}$ & $17.91 *$ & 9.72 \\
\hline $\begin{array}{l}\text { RBHP-61 x PRR-2007- } \\
2\end{array}$ & $18.64 *$ & 2.94 & $33.90 *$ & $13.14^{*}$ & $65.94 *$ & $42.91 *$ & $55.88 *$ & $47.22 *$ \\
\hline RBHP-61 × RBHP-900 & $-14.29 *$ & $-29.41 *$ & $-20.08 *$ & $-20.95^{*}$ & $-34.44 *$ & $-39.57 *$ & -1.49 & -8.33 \\
\hline $\begin{array}{l}\text { RBHP-107 } \times \text { PRR- } \\
\text { 2007-2 }\end{array}$ & $44.00^{*}$ & $44.00^{*}$ & $26.06^{*}$ & -1.08 & $60.99 *$ & $42.47 *$ & 2.86 & -5.26 \\
\hline $\begin{array}{l}\text { RBHP-107 } \times \text { RBHP- } \\
900\end{array}$ & $44.68 *$ & $36.00 *$ & $-25.81 *$ & $-32.95^{*}$ & $-22.55^{*}$ & $-30.63^{*}$ & 10.14 & 0.00 \\
\hline $\begin{array}{l}\text { RBHP-108 } \times \text { PRR- } \\
2007-2\end{array}$ & $16.98^{*}$ & 10.71 & $26.27 *$ & 1.27 & $29.53^{*}$ & $4.69 *$ & $-41.67 *$ & $-47.50 *$ \\
\hline $\begin{array}{l}\text { RBHP-108 } \times \text { RBHP- } \\
900\end{array}$ & $40.00 *$ & $25.00 *$ & $10.59 *$ & 2.64 & $-8.24 *$ & $-8.77 *$ & $43.66^{*}$ & $27.50^{*}$ \\
\hline
\end{tabular}




\begin{tabular}{|c|c|c|c|c|c|c|c|c|}
\hline \multirow[t]{2}{*}{ Crosses } & \multicolumn{2}{|c|}{ Seeds per pod } & \multicolumn{2}{|c|}{ 100-seed weight } & \multicolumn{2}{|c|}{ Pod length } & \multicolumn{2}{|c|}{ Yield per plant } \\
\hline & $\begin{array}{l}\text { Heterosis } \\
\text { over mid- } \\
\text { parent } \\
(\%)\end{array}$ & $\begin{array}{l}\text { Heterosis } \\
\text { over } \\
\text { better } \\
\text { parent } \\
(\%)\end{array}$ & $\begin{array}{l}\text { Heterosis } \\
\text { over mid- } \\
\text { parent } \\
(\%)\end{array}$ & $\begin{array}{l}\text { Heterosis } \\
\text { over } \\
\text { better } \\
\text { parent } \\
(\%)\end{array}$ & $\begin{array}{l}\text { Heterosis } \\
\text { over mid- } \\
\text { parent } \\
(\%)\end{array}$ & $\begin{array}{l}\text { Heterosis } \\
\text { over } \\
\text { better } \\
\text { parent } \\
(\%)\end{array}$ & $\begin{array}{l}\text { Heterosis } \\
\text { over mid- } \\
\text { parent } \\
(\%)\end{array}$ & $\begin{array}{l}\text { Heterosis } \\
\text { over } \\
\text { better } \\
\text { parent } \\
(\%)\end{array}$ \\
\hline $\begin{array}{l}\text { RBHP-36 } \times \\
\text { PRR-2007-2 }\end{array}$ & $12.66 *$ & 7.80 & $33.59 *$ & $29.48 *$ & $21.21 *$ & -4.38 & 1.04 & $-28.57 *$ \\
\hline $\begin{array}{l}\text { RBHP-36 x } \\
\text { RBHP-900 }\end{array}$ & 7.71 & 4.72 & $12.55 *$ & 6.85 & $25.98^{*}$ & 3.93 & $61.25^{*}$ & $30.41 *$ \\
\hline $\begin{array}{l}\text { RBHP-38 } \times \\
\text { PRR-2007-2 }\end{array}$ & $-16.91 *$ & $-27.12 *$ & $34.23^{*}$ & $49.84 *$ & -4.43 & $-25.89 *$ & $42.73^{*}$ & 1.77 \\
\hline $\begin{array}{l}\text { RBHP-38 } \times \\
\text { RBHP-900 }\end{array}$ & 0.95 & $-10.17 *$ & $13.96^{*}$ & $24.95^{*}$ & 5.64 & $-14.45^{*}$ & -5.37 & $-22.60 *$ \\
\hline $\begin{array}{l}\text { RBHP-43 } \times \\
\text { PRR-2007-2 }\end{array}$ & $26.87 *$ & 9.55 & $17.39^{*}$ & $21.37 *$ & $15.90 *$ & -4.80 & $52.35^{*}$ & 6.09 \\
\hline $\begin{array}{l}\text { RBHP-43 } \times \\
\text { RBHP-900 }\end{array}$ & 12.32 & -4.35 & $11.62 *$ & $13.20 *$ & -0.00 & $-13.85 *$ & 5.74 & -16.15 \\
\hline $\begin{array}{l}\text { RBHP-61 } \times \\
\text { PRR-2007-2 }\end{array}$ & $-10.95 *$ & $-16.58 *$ & 5.39 & 11.31 & $17.16^{*}$ & $-9.52 *$ & $121.00 *$ & $72.45^{*}$ \\
\hline $\begin{array}{l}\text { RBHP-61 } \times \\
\text { RBHP-900 }\end{array}$ & 5.72 & 0.59 & 9.04 & $13.01 *$ & $13.20 *$ & $-8.73^{*}$ & 19.98 & 10.18 \\
\hline $\begin{array}{l}\text { RBHP-107 } \\
\times \quad \text { PRR- } \\
2007-2\end{array}$ & $34.55^{*}$ & $24.72 *$ & $21.73^{*}$ & $29.92 *$ & $26.91 *$ & 1.19 & $30.94 *$ & -7.17 \\
\hline $\begin{array}{ll}\text { RBHP-107 } \\
\times \quad \text { RBHP- } \\
900 & \end{array}$ & $14.88 *$ & 4.89 & $-10.59 *$ & -6.35 & $30.99 *$ & $9.30 *$ & 6.32 & -13.69 \\
\hline $\begin{array}{l}\text { RBHP-108 } \\
\times \quad \text { PRR- } \\
2007-2\end{array}$ & $-26.98 *$ & $-29.10 *$ & 6.51 & 8.99 & 0.70 & $-20.39 *$ & 13.01 & $-21.33 *$ \\
\hline \begin{tabular}{ll}
\multicolumn{2}{l}{ RBHP-108 } \\
$\times$ & RBHP- \\
900 &
\end{tabular} & 1.34 & -0.00 & $19.82 *$ & $20.24 *$ & $28.38 *$ & 6.15 & -10.07 & $-28.72 *$ \\
\hline
\end{tabular}


Table.3 Mean performance of parents and their hybrids for different traits

\begin{tabular}{|c|c|c|c|c|c|c|c|c|c|c|c|}
\hline Traits & $\begin{array}{c}\text { Days to } \\
\text { flowering }\end{array}$ & $\begin{array}{l}\text { Days to } \\
\text { Maturity }\end{array}$ & $\begin{array}{c}\text { Plant } \\
\text { height } \\
\text { (cm) }\end{array}$ & $\begin{array}{l}\text { Branches } \\
\text { per plant }\end{array}$ & $\begin{array}{c}\text { Pods } \\
\text { per } \\
\text { plant }\end{array}$ & $\begin{array}{c}\text { Clusters } \\
\text { per plant }\end{array}$ & $\begin{array}{l}\text { Pods } \\
\text { per } \\
\text { cluster }\end{array}$ & $\begin{array}{l}\text { Seeds } \\
\text { per } \\
\text { pod }\end{array}$ & $\begin{array}{c}100 \text { seed } \\
\text { weight }\end{array}$ & $\begin{array}{l}\text { Pod } \\
\text { length } \\
\text { (cm) }\end{array}$ & $\begin{array}{c}\text { Yield } \\
\text { per } \\
\text { plant }\end{array}$ \\
\hline $\begin{array}{l}\text { RBHP-36 X } \\
\text { PRR-2007-2 }\end{array}$ & 66.33 & 96.00 & 77.23 & 1.33 & 31.33 & 15.13 & 2.20 & 7.00 & 6.87 & 9.91 & 8.09 \\
\hline $\begin{array}{l}\text { RBHP-36X } \\
\text { RBHP-900 }\end{array}$ & 74.00 & 128.00 & 110.67 & 2.40 & 46.67 & 21.33 & 3.14 & 6.80 & 5.67 & 10.77 & 14.78 \\
\hline $\begin{array}{l}\text { RBHP-38 X } \\
\text { PRR-2007-2 }\end{array}$ & 67.67 & 96.33 & 79.91 & 2.27 & 31.25 & 20.14 & 2.33 & 5.73 & 7.95 & 8.05 & 11.20 \\
\hline $\begin{array}{l}\text { RBHP-38X } \\
\text { RBHP-900 }\end{array}$ & 69.33 & 105.00 & 69.91 & 1.40 & 29.00 & 12.87 & 2.47 & 7.07 & 6.63 & 9.30 & 8.52 \\
\hline $\begin{array}{l}\text { RBHP-43 X } \\
\text { PRR-2007-2 }\end{array}$ & 68.00 & 106.00 & 119.06 & 2.20 & 44.59 & 21.20 & 2.50 & 6.50 & 6.44 & 8.87 & 12.67 \\
\hline $\begin{array}{l}\text { RBHP-43X } \\
\text { RBHP-900 }\end{array}$ & 67.00 & 100.00 & 101.87 & 2.27 & 32.66 & 13.87 & 2.63 & 5.87 & 6.00 & 8.02 & 10.01 \\
\hline $\begin{array}{l}\text { RBHP-61 X } \\
\text { PRR-2007-2 }\end{array}$ & 71.00 & 110.33 & 116.37 & 2.33 & 41.60 & 24.27 & 3.53 & 5.67 & 5.90 & 9.95 & 14.42 \\
\hline $\begin{array}{l}\text { RBHP-61X } \\
\text { RBHP-900 }\end{array}$ & 69.33 & 105.00 & 95.26 & 1.60 & 29.07 & 12.17 & 2.20 & 6.83 & 5.99 & 10.03 & 9.21 \\
\hline $\begin{array}{l}\text { RBHP-107 } \\
\text { X PRR- } \\
2007-2\end{array}$ & 70.00 & 112.00 & 128.99 & 2.40 & 44.07 & 22.70 & 2.40 & 7.40 & 6.89 & 10.19 & 10.41 \\
\hline $\begin{array}{l}\text { RBHP-107X } \\
\text { RBHP-900 }\end{array}$ & 69.00 & 134.67 & 76.51 & 2.27 & 29.87 & 13.97 & 2.53 & 6.43 & 4.97 & 11.00 & 9.68 \\
\hline $\begin{array}{l}\text { RBHP-108 } \\
\text { X PRR- } \\
2007-2\end{array}$ & 80.00 & 113.67 & 70.83 & 2.07 & 42.53 & 20.83 & 1.40 & 4.47 & 5.78 & 8.20 & 9.40 \\
\hline $\begin{array}{l}\text { RBHP-108 } \\
\text { X RBHP- } \\
900\end{array}$ & 68.33 & 101.00 & 92.34 & 2.33 & 43.11 & 18.37 & 3.40 & 6.30 & 6.38 & 10.93 & 8.52 \\
\hline RBHP-36 & 79.67 & 121.00 & 125.88 & 1.73 & 37.65 & 14.00 & 2.33 & 6.49 & 5.30 & 10.36 & 11.33 \\
\hline RBHP-38 & 80.33 & 115.33 & 111.22 & 1.40 & 35.98 & 15.32 & 2.37 & 7.87 & 6.86 & 10.87 & 11.00 \\
\hline RBHP-61 & 79.33 & 111.67 & 111.25 & 2.27 & 36.77 & 16.98 & 2.40 & 6.79 & 6.23 & 10.99 & 8.36 \\
\hline RBHP-107 & 80.67 & 107.67 & 107.17 & 1.67 & 44.55 & 15.93 & 2.53 & 5.07 & 6.34 & 10.07 & 11.22 \\
\hline RBHP-108 & 83.67 & 115.67 & 114.43 & 1.87 & 42.00 & 19.90 & 2.67 & 6.30 & 5.88 & 10.30 & 11.95 \\
\hline PRR-2007-2 & 51.00 & 90.33 & 64.17 & 1.67 & 25.37 & 12.27 & 2.13 & 5.93 & 4.98 & 5.99 & 4.69 \\
\hline RBHP-900 & 63.33 & 100.67 & 82.54 & 1.47 & 35.97 & 20.13 & 2.07 & 6.13 & 4.77 & 6.73 & 7.00 \\
\hline RBHP-43(C) & 79.67 & 113.33 & 118.33 & 1.07 & 34.84 & 16.46 & 2.40 & 4.31 & 5.99 & 9.31 & 11.94 \\
\hline Mean & 71.88 & 109.18 & 98.69 & 1.90 & 36.94 & 17.39 & 2.48 & 6.20 & 6.09 & 9.49 & 10.22 \\
\hline CD & 2.3 & 3.7 & 9.6 & 0.3 & 2.2 & 0.8 & 0.4 & 0.8 & 0.7 & 0.9 & 2.2 \\
\hline $\mathrm{CV}$ & 1.95 & 2.07 & 5.90 & 8.87 & 3.68 & 2.83 & 9.84 & 7.79 & 6.06 & 5.34 & 13.13 \\
\hline
\end{tabular}


Table.4 Analysis of variance for parents and hybrids

\begin{tabular}{|c|c|c|c|c|c|c|c|c|c|}
\hline $\begin{array}{l}\text { Source of } \\
\text { variation }\end{array}$ & & Replication & Parents & Lines & Testers & $\begin{array}{c}\text { Lines vs. } \\
\text { testers }\end{array}$ & Crosses & $\begin{array}{c}\text { Parents vs. } \\
\text { Hybrid }\end{array}$ & Error \\
\hline Traits & df & 2 & 7 & 5 & 1 & 1 & 11 & 1 & 38 \\
\hline $\begin{array}{l}\text { Days to } 50 \% \\
\text { flowering }\end{array}$ & & 3.51 & $389.75^{*}$ & $7.68 *$ & $228.16^{*}$ & $2461.68 *$ & $41.87 *$ & $319.225^{*}$ & 1.972 \\
\hline $\begin{array}{l}\text { Days to } 75 \% \\
\text { maturity }\end{array}$ & & 1.71 & $288.18 *$ & $59.68 *$ & $160.16^{*}$ & $1558.68 *$ & $427.57 *$ & $3.025^{*}$ & 5.102 \\
\hline Plant height & & 95.16 & $1265.73^{*}$ & $\begin{array}{c}131.44 \\
*\end{array}$ & $505.81 *$ & $7697.09 *$ & $1251.85^{*}$ & $1289.284^{*}$ & 33.916 \\
\hline $\begin{array}{l}\text { Branches per } \\
\text { plant }\end{array}$ & & 0.07 & $0.37 *$ & $0.50 *$ & 0.060 & 0.045 & $0.46^{*}$ & 0.045 & 0.029 \\
\hline Pods per plant & & 0.64 & $95.77 *$ & $43.29 *$ & $168.540 *$ & $285.44^{*}$ & $150.49 *$ & $3.672 *$ & 1.851 \\
\hline $\begin{array}{l}\text { Clusters per } \\
\text { plant }\end{array}$ & & 0.41 & $21.74 *$ & $11.83^{*}$ & $92.826^{*}$ & 0.24 & $53.821^{*}$ & $41.391 *$ & 0.243 \\
\hline Pods per cluster & & 0.44 & 0.11 & 0.04 & 0.006 & $0.55^{*}$ & $1.006^{*}$ & $0.571^{*}$ & 0.059 \\
\hline Seeds per pod & & 0.14 & $3.46^{*}$ & $4.82 *$ & 0.060 & 0.05 & $1.945^{*}$ & $0.738^{*}$ & 0.234 \\
\hline 100 seed weight & & 1.08 & $1.56^{*}$ & $0.81 *$ & 0.066 & $6.79 *$ & $1.717^{*}$ & $3.524 *$ & 0.136 \\
\hline Pod length (cm) & & 1.46 & $10.96 *$ & $1.09 *$ & $0.836^{*}$ & $70.44^{*}$ & $3.633^{*}$ & $1.080 *$ & 0.256 \\
\hline Yield per plant & & 36.31 & $21.84 *$ & $5.34 *$ & $7.990 *$ & $118.21 *$ & $15.322 *$ & $11.403^{*}$ & 1.801 \\
\hline
\end{tabular}

Based on mean values (Table 3) it is concluded that among all the hybrids, aamong the parents, RBHP-108 (11.95 g), RBHP-43 (11.94g), RBHP-36 (11.33g), RBHP-107 (11.22g) and RBHP-38 (11.00g) were the highest yielders whereas among the hybrids, RBHP-36 x RBHP-900 (14.78g), RBHP61x2007-2 (14.42g) and RBHP-43x2007-2 $(12.67 \mathrm{~g})$ recorded highest grain yield per plant. Two crosses RBHP-36x2007-2 (96 days) and RBHP-38x2007-2 (96.33days) were found to be significantly early, determinate with good per plant yield among all the crosses which could be exploited through heterosis breeding programme in future to develop high yielding early maturing and determinate varieties of ricebean.

\section{References}

Ebert Andreas, W 2014. Potential of underutilized traditional vegetables and legume crops to contribute to food and nutritional security, income and more sustainable production systems.
Sustainability 6: 319-335

Kempthorne, O., 1957. An Introduction to Genetic Statistics. John Wiley and Sons, New York pp Panse VG and Sukhatme PV. 1984. Statistical Methods for Agricultural Workers. Indian Council of Agricultural Research, New Delhi p 381: 458- 471 Lakshmana, D., Reddy BG and Ramesh S 2007. Heterosis studies in rice bean (Vign umbellata (Thunb.) Ohwi and Ohashi). Legume Research- An International Journal, 30(3), pp.209211.

Sharma, A., Singh MRK and Singh NB 1998. Heterosis and combining ability for grain yield and its components in ricebean (Vigna umbellata (thunb) Ohwi and Ohashi). Indian Journal of Hill Farming, 11(1\&2): 27-33.

Vaidya, GB., Chauhan DA, Narwade AV, Kale BH and Pandya MM 2016. Heterosis for yield and yield attributing characters in rabi mungbean [Vigna radiata (L.) 
Wilczek]. Legume Research 39: 657664.

Thamodharan, G., Geetha S and Ushakumari

$\mathrm{R}$ 2016. Studies on heterosis in black gram [Vigna mungo (L.) Hepper]. Indian Journal of Agricultural Research 50: 406-413

\section{How to cite this article:}

Neelam Bhardwaj, Tanuja Kapoor and Sanchit Thakur. 2019. Heterosis Studies for Seed Yield and Earliness in Intra-specific Hybrids of Ricebean [Vigna Umbellata (Thunb.) Ohwi and Ohashi] an under Utilized Pulse. Int.J.Curr.Microbiol.App.Sci. 8(04): 1012-1019. doi: https://doi.org/10.20546/ijcmas.2019.804.117 